Ryan, F. J., Fried, P. \& Schwartz, M. (1954). J. gen. Microbiol. 11, 380-393.

\title{
Nuclear Segregation and the Growth of Clones of Bacterial Mutants induced by Ultraviolet Light
}

\author{
By F. J. RYAN, PHYLLIS FRIED AND MIRIAM SCHWARTZ \\ Department of Zoology, Columbia University, New York 27
}

SUMMARY: Histidine-independent $\left(\mathrm{h}^{+}\right)$mutants induced in histidine-requiring $\left(h^{-}\right)$cultures by ultraviolet light have a delay in the onset of logarithmic increase that is about two generations longer than the delay shown by marked unirradiated $\mathrm{h}^{+}$bacteria present at the same time. This extra delay is interpreted as being due to the segregation of one from four nuclei which are present, on the average, in growing $h^{-}$organisms. The same assumption accounts for the extra delay observed in spontaneous $\mathrm{h}^{+}$mutants. These and other results are discussed in relation to the site of mutation and to the various types of delay that can retard the onset of growth of a mutant clone. It is concluded that in the mutation from $h^{-}$to $h^{+}$, the $h^{+}$condition is dominant in the heterocaryon, that whatever phenotypic delay exists is short, and that cell division is not required to pass through it.

In the previous paper (Ryan \& Wainwright, 1954) evidence was presented that led to the conclusion that growing Escherichia coli organisms have their genetic material represented at least 4 times, on the average, in each bacterium. In histidine-dependent $\left(\mathrm{h}^{-}\right)$bacteria, the mutation leading to a histidine-independent condition $\left(\mathrm{h}^{+}\right)$occurs independently among members of each set. Thus bacteria are produced which are initially heterocaryotic. Only after growth and division does the heterocaryon segregate its nuclei so that a homocaryotic mutant offspring is produced and only then does the clone of mutants increase by doubling. This delay is referred to as segregation lag and, in the system studied, lasted for a minimum of two generations. The evidence came from the overall rate of increase of mutants in growing populations of mutating parental bacteria and was therefore indirect. It seemed desirable to perform experiments with populations in which a large crop of new mutants had just been induced. The number present would not be expected to increase until they had become homocaryotic by the segregation of their non-mutant nuclei during growth. The length of the delay would be a function of the average number of nuclei per bacterium according to the relation:

$$
\text { generations of segregation } \operatorname{lag}=\frac{\log \text { no. of nuclei/bacterium }}{\log 2} .
$$

This possibility was approached directly by exposing $\mathbf{h}-$ bacteria to ultraviolet light until more than $90 \%$ of the $\mathrm{h}^{+}$mutants present were induced mutants. The culture was then spread on the surface of agar devoid of histidine where only the $\mathrm{h}^{+}$mutants were able to grow and form colonies. From time to time the surfaces were re-spread in a way that separated all of the growing bacteria present. Only after segregation lag was over did the number of $\mathrm{h}^{+}$ colonies increase. Since the irradiated culture had to be washed in order to remove histidine from the $h^{-}$parents and prevent their growth, and had to be 
plated from liquid on to an agar surface, a physiological lag was induced. To measure the length of the delay from these causes, cultures of $\mathrm{h}^{+} \mathrm{lac}^{-}$bacteria that could not ferment lactose were spread with the $\mathrm{h}^{-}$bacteria at the beginning of the experiment. The difference between the delay in increase of numbers of new $h^{+}$mutants and added marked $h^{+}$bacteria was taken as a measure of the length of segregation lag because the added $\mathrm{h}^{+}$lac- mutants were already homocaryotic for $\mathrm{h}^{+}$nuclei. The results of these experiments were consistent with the existence of an average of four nuclei per growing bacterium. Furthermore, when similar procedures were applied to cultures containing spontaneous mutants, only a fraction of which were less than two generations old, the results led to the same conclusion.

\section{MATERIALS AND METHODS}

The strains and basic techniques used in these experiments have been described elsewhere (Ryan \& Wainwright, 1954; Ryan, 1954). Ultraviolet light was used to induce new histidine-synthesizing $\left(\mathrm{h}^{+}\right)$mutants because they were not found after treatment of $\mathrm{h}^{-}$bacteria with such non-toxic mutagens as caffein, theophylline or $\mathrm{MnCl}_{2}$ (the $\mathrm{h}^{-}$locus is apparently stable in the presence of some mutagens; Demerec \& Cahn, 1953). Appropriate inoculations of a histidineless strain $\left(\mathrm{h}^{-}\right)$of Escherichia coli were made into $1 \mathrm{l}$. flasks containing $250 \mathrm{ml}$. lots of chemically defined salt medium with $0.05 \%(\mathrm{w} / \mathrm{v})$ glucose and a concentration of histidine which yielded optimal growth. After overnight agitation at $37^{\circ}$ the bacteria were in the logarithmic phase of growth and at a concentration of $c .2 \times 10^{8} \mathrm{organisms} / \mathrm{ml}$. The cultures were washed once by centrifugation in $0.9 \% \mathrm{NaCl}$, resuspended in one-tenth of the original volume and distributed to Petri dishes in $10 \mathrm{ml}$. lots. In this condition they were illuminated while being rotated at a distance of $14 \mathrm{~cm}$. from a Westinghouse 'Sterilamp'. The suspensions were then pooled, washed once in saline and resuspended so as to make a total concentration of one-hundred-fold. All operations after the irradiation were performed in the yellow light of a G.E. 'Bugaway' lamp to prevent photo-reactivation.

The cultures were diluted when necessary and spread by the use of a sterile glass rod and a rotating stand on to the surfaces of agar plates containing or not containing histidine. After low doses of ultraviolet light $(<40 \mathrm{sec}$.) the resulting colonies were easily countable after 2 days incubation at $37^{\circ}$; after higher doses of irradiation it was necessary to wait 3 days before all of the induced $\mathrm{h}^{+}$mutant colonies developed. This is because the $\mathrm{h}^{+}$mutants induced by high doses of ultraviolet light appear in a fraction of the population that has a very long induced delay in the onset of division (Ryan, 1954). Fig. 1 shows the results obtained with a variety of doses of ultraviolet light. As the survival drops to $10^{-5}$ the frequency of induced $h^{+}$mutants among the survivors rises to between $10^{-4}$ and $10^{-3}$. The mutant increase curve is extremely steep after an initial lag and this may account, in part, for the failure of Lieb (1951) to induce a considerable number of $\mathrm{h}^{+}$mutants in this strain with doses of ultraviolet light which left as many as $10^{-3}$ survivors. It is interesting to note that the killing curve of these $\mathrm{h}^{-}$bacteria is exponential down to $c .10^{-4}$ 
or $10^{-5}$ under the conditions used. Since the organisms in growing cultures contain an average of more than one nuclear body, it may be concluded that nuclear inactivation is not the main cause of bacterial inactivation. An $\mathrm{h}^{-}$ survivor of 120 sec. of irradiation, when isolated, grown and re-irradiated, gave survival and induced mutant curves indistinguishable from those of its parent. It does not seem probable, therefore, that the break in the survival curve shown in Fig. 1 was due to a small fraction of the population of bacteria being genetically resistant to ultraviolet light.

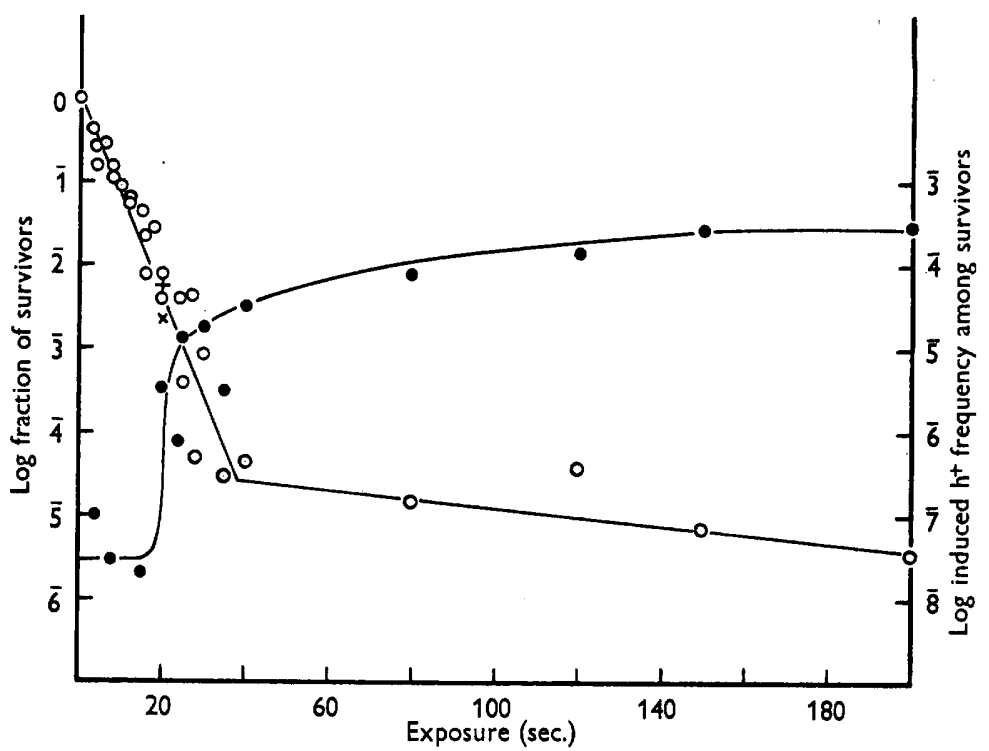

Fig. 1. Survival of, and induced $h^{+}$mutant frequency among, $h^{-}$bacteria removed from the condition of logarithmic growth and exposed to ultraviolet light for various periods of time. The left ordinate measures the $\log _{10}$ of the fraction of survivors (open circles), the ordinate on the right, the $\log _{10}$ of the frequency of induced mutants among the survivors (black circles); and the abscissa, the length of exposure to ultraviolet light in seconds. The data for 20 sec. are the averages of 15 experiments and were therefore weighted in drawing the curves (the black circle that lies out of relation to its mates is based upon a single determination). The average frequency of spontaneous $h^{+}$mutants was $3.4 \times 10^{-7}$. The $x$ is for the survival of an $h^{+}$mutant previously secured from the $h^{-}$; the + is for an $h^{+}$lac $^{-}$strain derived from the $h^{-}$in two steps.

For the experiments to be reported in this paper a dose of 20 sec., yielding an average survival of $4 \times 10^{-3}$, was chosen. This dose seemed suitable because: (i) it usually resulted in more than $90 \%$ of the $\mathrm{h}^{+}$mutants being induced and new; (ii) it did not produce the extremely long extra physiological delay in the onset of growth of the induced $\mathrm{h}^{+}$mutants found after high doses of ultraviolet light (Ryan, 1954); (iii) it did not appear to inactivate the washed bacteria in such a way as to enable them to decompose and yield appreciable histidine to the medium. Fig. 2 shows the result of spreading $\mathbf{0 . 2} \mathrm{ml}$. of an irradiated culture on a series of agar plates devoid of histidine and, at intervals thereafter, washing the suspensions from the surfaces by scrubbing in two successive lots of $5 \mathrm{ml}$. of added sterile saline with the aid of a sterile glass rod 
and a turntable. The two lots were pooled, washed and plated on agar with and without histidine for differential assay of viable $h^{+}$and $h^{-}$bacteria. The washing-off procedure did not result in a detectable loss of bacteria. In three experiments designed to test the efficiency of this procedure an average of $90 \%$ of the $\mathrm{h}^{-}$and $114 \%$ of the $\mathrm{h}^{+}$bacteria estimated to have been on the plates were recovered by washing off.

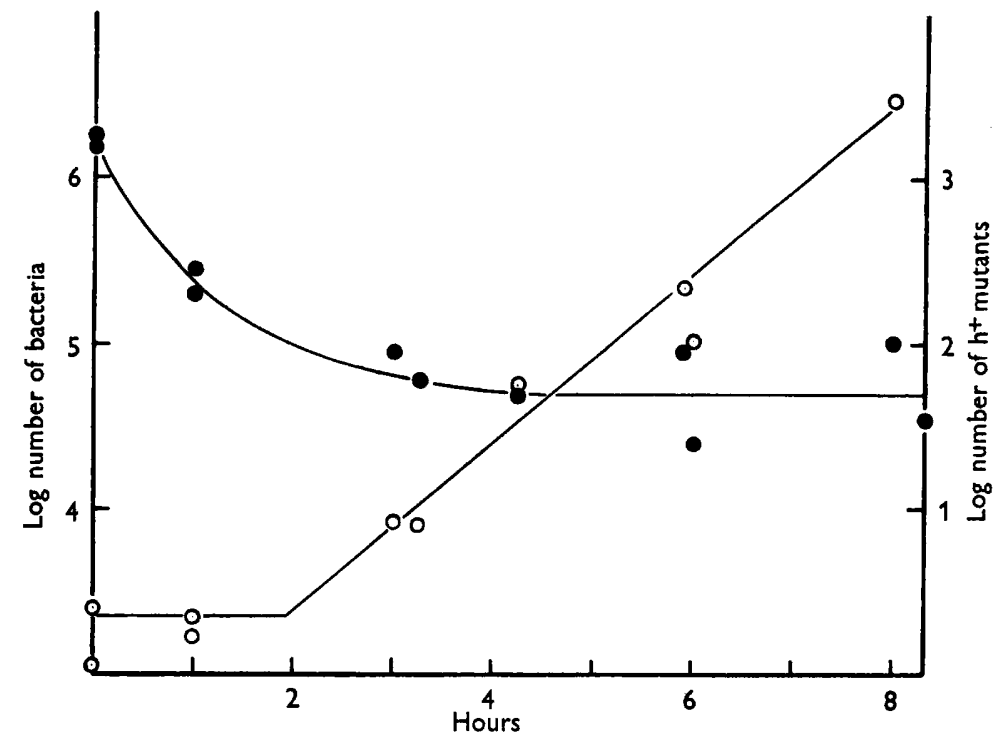

Fig. 2. The behaviour of irradiated $h^{-}$bacteria (black circles) and of the $h^{+}$mutants (open circles) induced among them after being spread on agar devoid of histidine. The left ordinate measures the $\log _{10}$ of the number of viable $h^{-}$bacteria/plate; the ordinate on the right, the $\log _{10}$ of the number of viable $h^{+}$bacteria/plate; and the abscissa, the time in hours at which they were washed from the plates for assay. The data come from 2 experiments where the average survival was $6 \cdot 3 \times 10^{-4}$ but where only $56 \%$ of the mutants present were induced. The generation time for the $h^{+}$bacteria during logarithmic growth was 35 min.

It can be seen from Fig. 2 that the surviving $h^{+}$bacteria grew normally after a short lag (a generation time of about $35 \mathrm{~min}$. instead of $55 \mathrm{~min}$. was usually found during logarithmic growth of cultures surviving an irradiation of $20 \mathrm{sec}$; this is probably due to the release of some stimulating materials by the inactivated organisms). The surviving $h^{-}$bacteria did not find histidine on the plates with which to grow and divide but, as a matter of fact, underwent considerable death upon incubation.* After more than $10 \mathrm{hr}$. they appeared to autolyse and release a little histidine; after $22 \mathrm{hr}$. the numbers of viable $\mathrm{h}^{-}$ bacteria on the plates in the experiments shown in Fig. 2 had increased from

* Death of irradiated bacteria of a similar sort was discovered by Lieb (1951). She found that a large fraction of irradiated bacteria quickly died when suspended in liquid medium but survived when agar was present. The phenomenon was called post-irradiation death and has been confirmed by us. Furthermore, although it does not occur on agar containing histidine it does occur on agar devoid of histidine (Fig. 2) provided that the bacteria have not been so heavily irradiated that histidine is released by the autolysis of inactivated bacteria (Ryan, 1954). 
$1 \cdot 9 \times 10^{6}$ to $7 \cdot 4 \times 10^{6}$. But this increase occurs far too late to have any influence on the initial increase in number of $h^{+}$bacteria.

In most of the experiments to be reported the bacteria were not washed from the plates but were re-spread at some later time in $\mathbf{0 . 2} \mathrm{ml}$. added sterile saline. Since there was no histidine on the plates, only the $\mathrm{h}^{+}$mutants were able to form colonies: it was in the course of this growth that the clones were separated by the re-spreading procedure. Evidence that the sister bacteria were efficiently separated comes from the observation of the normal logarithmic growth curves resulting; for example, unirradiated $h^{+}$bacteria were observed to have a generation time of $51 \mathrm{~min}$. by this method as compared to 50-55 min. by other methods but under similar conditions. Furthermore, the number of $\mathrm{h}^{+}$mutant bacteria present on the plates at any time, as determined by the spreading or the washing-off techniques, was the same.

In order to interpret the length of the delay of the new $\mathrm{h}^{+} \mathrm{lac}^{-}$or $\mathrm{h}^{+} \mathrm{lac}^{+}$ mutants, $h^{+}$bacteria from established stocks and carrying either a lac ${ }^{+}$ (lactose-fermenting) or a lac- (non-lactose-fermenting) marker were added in appropriate amounts to the irradiated $\mathrm{h}^{-}$cultures just before the latter were first spread. The combinations used are described in Table 1. All of the strains used had, in tenfold concentrations and when taken as they were from the growing condition, the same sensitivity to ultraviolet light (Fig. 1).

Table 1. Variations in the mixtures introduced on the surfaces of plates devoid of histidine and respread

\begin{tabular}{|c|c|c|c|c|c|}
\hline Mixture & Strains & $\begin{array}{l}\text { Volume } \\
\text { grown in } \\
\text { (ml.) }\end{array}$ & Irradiated & $\begin{array}{c}\text { Final } \\
\text { concentration }\end{array}$ & $\begin{array}{c}\text { Results } \\
\text { described in }\end{array}$ \\
\hline $\mathbf{A}$ & $\begin{array}{l}\mathrm{h}^{-} \mathrm{lac}^{+} \\
\mathrm{h}^{+} \mathrm{lac}^{-}\end{array}$ & 250 & $\begin{array}{l}\text { Yes } \\
\text { Yes }\end{array}$ & $100 \times$ & Fig. 3 \\
\hline B & $\begin{array}{l}\mathbf{h}^{-} \mathbf{l a c}^{+} \\
\mathbf{h}^{+} \mathbf{l a c}^{-}\end{array}$ & 250 & $\begin{array}{l}\text { Yes } \\
\text { No }\end{array}$ & $100 \times$ & Fig. 4 \\
\hline C & $\begin{array}{l}h^{+} \text {lac }^{+} \\
h^{+} \text {lac }^{-}\end{array}$ & 100 & $\begin{array}{l}\text { Yes } \\
\text { No }\end{array}$ & $10 \times$ & Fig. 5 \\
\hline D & $\begin{array}{l}\mathbf{h}^{+} \text {lac }^{+} \\
\mathbf{h}^{+} \text {lac }^{-}\end{array}$ & 100 & $\begin{array}{l}\text { No } \\
\text { Yes }\end{array}$ & $10 \times$ & Text \\
\hline $\mathbf{E}$ & $\begin{array}{l}h^{-} \text {lac }^{+} \\
h^{+} \text {lac }^{-}\end{array}$ & 100 & $\begin{array}{l}\text { No } \\
\text { No }\end{array}$ & $1 \times$ & Fig. 6 \\
\hline
\end{tabular}

Experiments designed to test for the possibility of suppression of the growth of $\mathrm{h}^{+}$mutants on the crowded plate surfaces were performed. First, suspensions of $h^{-}$bacteria were irradiated for 20 sec., concentrated as usual and then spread on plates devoid of histidine in dilutions ranging around that used routinely in the re-spreading experiments. The numbers of $\mathrm{h}^{+}$colonies found were appropriate for the dilution. Secondly, in nine experiments where an estimated average of 14 marked $h^{+}$bacteria had been added to plates containing irradiated $\mathrm{h}^{-}$or $\mathrm{h}^{+}$organisms, 19 marked $\mathrm{h}^{+}$colonies were recovered on the average. Hence with the method used the results were not influenced by suppression of $\mathrm{h}^{+}$colonies nor by mutations occurring on the plates. 
Furthermore, in ten experiments where an estimated average of 9 marked $\mathrm{h}^{+}$ bacteria were added to plates devoid of histidine but containing the unirradiated $\mathrm{h}$ - suspensions used in the studies of the delay in the growth of spontaneous $h^{+}$mutants, 11 marked $h^{+}$colonies were recovered on the average; and in four experiments where an estimated average of 12 marked survivors of a suspension of $\mathrm{h}^{+}$bacteria irradiated for 120 sec. were added to plates containing $\mathrm{h}^{-}$organisms irradiated to the same extent, an average of 10 marked $\mathrm{h}^{+}$ colonies was found. Hence, the conditions used in the experiments reported here and elsewhere (Ryan, 1954) did not suppress $\mathrm{h}^{+}$colonies.

In all spreading experiments the increase in numbers of new and added $\mathrm{h}^{+}$ mutants was followed by the use of two series of plates all devoid of histidine. On one series $0.1 \mathrm{ml}$. of only the irradiated $\mathrm{h}^{-}\left(\right.$or $\left.\mathrm{h}^{+}\right)$bacteria was spread; on the other, the same bacteria and the added mutants were spread together. On the latter plates discrimination between the marked and unmarked (lac ${ }^{-}$ and $\mathrm{lac}^{+}$) bacteria was achieved with eosin methylene-blue agar by the replication technique of Lederberg \& Lederberg (1952). The numbers of the new (or, in some cases, irradiated) $\mathrm{h}^{+}$bacteria were taken as the averages of the colony counts on the first series of plates and the counts of the proper lac type on the replicate plates. The numbers of the added component were taken directly from the replicate plates; when these were too crowded for accurate reading, the numbers were taken as the difference between the counts on plates of the first and second series.

Cytologica lstudies were made on preparations of growing $h^{-}$bacteria taken from defined medium + histidine. The procedures adopted were essentially those of Robinow (1945) and involved Giemsa stain.

\section{RESULTS}

Fig. 3 shows the growth curves for $\mathrm{h}^{+}$mutants arising in irradiated $\mathrm{h}^{-}$lac ${ }^{+}$ cultures and of $\mathrm{h}^{+} \mathrm{lac}^{-}$mutants that were irradiated to the same survival and mixed with them. The lag period for both mutant types was the same and lasted for a little more than $2 \mathrm{hr}$. or a time equivalent to about 4 generations. Since both of the $\mathrm{h}^{+}$mutants were irradiated it is very likely that only one surviving nucleus was present/surviving bacterium.* The segregation of viable from inactive $h^{+}$nuclei in the $h^{+}$lac $^{-}$strain and of viable $h^{+}$from inactive $h^{-}$ nuclei in the new mutants is probably the cause of the equal delays in growth. Similarly, in two experiments, where irradiated $h^{-}$cultures were spread on plates containing histidine and then washed off for a differential assay of $h^{+}$ mutants and $h^{-}$parents, no significant difference could be observed in the

* These $\mathrm{h}^{+}$bacteria had been exposed to a dose of ultraviolet light on the exponential part of the killing curve (Fig. 1). As mentioned earlier, this curve is not a function of the cumulative inactivation of the nuclei present. Nonetheless, when more than $99 \%$ of the bacteria have been killed it seems probable that three of the four nuclei may have been inactivated among the survivors. The relation between nuclear inactivation and cell killing is clearly complex; there is not even reason to believe it is the same in the spores of Neurospora (Atwood, unpublished) where there are several nuclei in a common cytoplasm, and in growing Escherichia coli, where cell walls eventually separate the nuclei originally present. 
times of onset of growth. The proper control thus appeared to be added $\mathrm{h}^{+} \mathrm{lac}^{-}$mutants which had not been irradiated.

Such an experiment is shown in Fig. 4 which describes the growth of $\mathrm{h}^{+}$ mutants produced by the irradiation of an $\mathrm{h}^{-\mathrm{lac}^{+}}$culture and of unirradiated $\mathrm{h}^{+}$lac- bacteria which were spread on the same plates at the beginning of the experiment. The difference in the times of onset of growth is estimated as equal to $\mathbf{1 . 8}$ generations, or $\mathbf{1 . 9}$ generations if the extra delays in the three individual component experiments are separately determined and averaged. These estimated delays are not significantly different from that of 2 generations expected for the segregation of four nuclei.

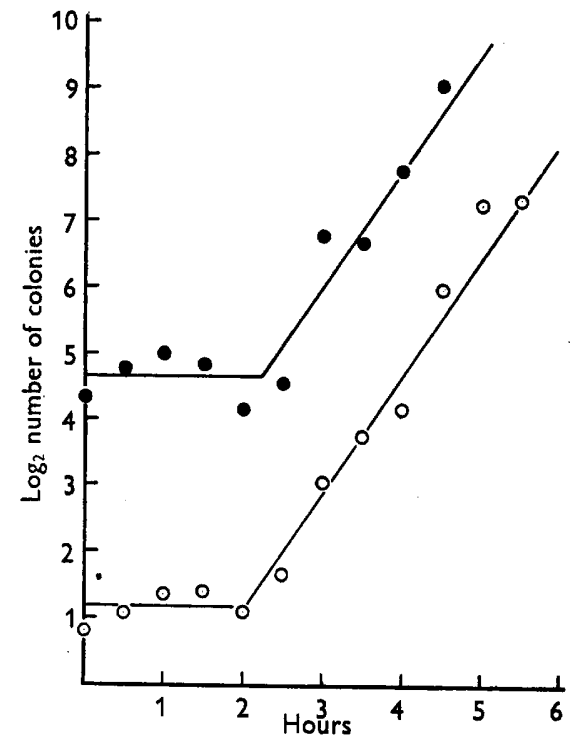

Fig. 3

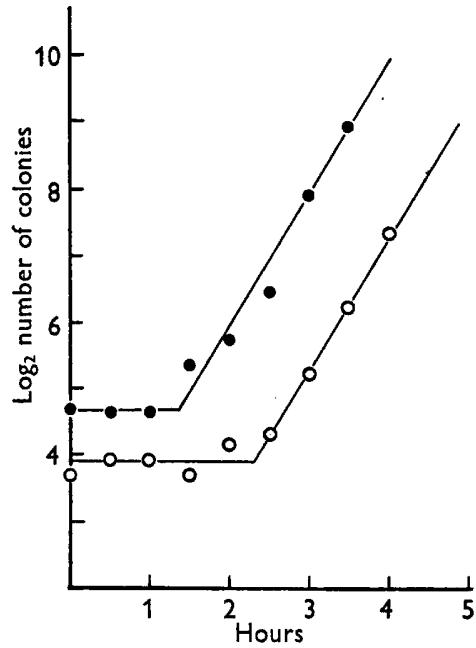

Fig. 4

Fig. 3. The growth of $h^{+}$mutants (open circles) found among irradiated $h^{-}$lac ${ }^{+}$bacteria spread on the surfaces of agar devoid of histidine and of irradiated $h^{+}$lac- bacteria (black circles) that had been added to them. The ordinate measures the $\log _{2}$ of the number of colonies/plate; the abscissa, the time (hr.) at which the plates were respread. The data are the averages of 2 experiments where the average survival was $1 \cdot 3 \times 10^{-8}$ and where $98 \%$ of the mutants present were induced. The generation time during logarithmic growth was $34 \mathrm{~min}$.

Fig. 4. The growth of $h^{+}$mutants (open circles) found among irradiated $h^{-}$lac $^{+}$bacteria spread on the surfaces of agar devoid of histidine and of unirradiated $h^{+}$lac ${ }^{-}$(black circles) that had been added to them. The ordinate measures the $\log _{2}$ of the number of colonies/plate; the abscissa, the time (hr.) at which the plates were respread. The data are the averages of 3 experiments where the average survival was $1.9 \times 10^{-2}$ and where $99 \%$ of the mutants present were induced. The generation time during logarithmic growth was $30 \mathrm{~min}$.

When unirradiated $\mathrm{h}^{+}$lac- bacteria were added to irradiated $\mathrm{h}^{+} \mathrm{lac}^{+}$cultures the results shown in Fig. 5 were obtained. The irradiated mutants had an extra lag of 1.6 generations as calculated from the average curve or of 1.8 generations when the extra lags in the individual component experiments 
were determined and averaged. In one experiment involving the mixture of irradiated $\mathrm{h}^{+} \mathrm{lac}^{-}$and unirradiated $\mathrm{h}^{+} \mathrm{lac}^{+}$bacteria, the lag of the irradiated bacteria was 1.3 generations longer than that of the unirradiated. In view of the errors involved in this sort of experiment and the observed variability $(\mathbf{1} \cdot 3-2 \cdot 3$ generations) it is concluded that the lac marker is neutral and that the extra delay in the onset of growth of new mutants is close to 2 generations.

When the length of exposure to ultraviolet light is increased to $120 \mathrm{sec}$. so that survival is reduced to $c .10^{-5}$, an extremely long delay lasting sometimes for more than 30 generations is induced in new $h^{+}$mutants (Ryan, 1954). The 2-generation delay following a 20 sec. exposure to ultraviolet light could conceivably be of this physiological sort. The physiological delay after high doses of ultraviolet light affected the mutant, not the parent, and was extremely variable, while, on the other hand, the 2-generation extra delay is consistent and unspecific. Furthermore, the eventual onset of growth is of an abruptness not expected if the delay had a physiological cause or were due to induced irregularities in the onset of division of individual members of the population (Newcombe \& Scott, 1949). Perhaps the strongest argument for nuclear segregation as the cause of the 2-generation delay is that the assumption of an average of four nuclei accounts not only for it, but also for delays in growth of spontaneous mutants (Ryan \& Wainwright, 1954). This is also true when spontaneous mutants are studied by the same methods that were used for the induced mutants.

Because of the growth of earlier mutants, the proportion of spontaneous mutants less than 2 generations old continually decreases during the growth of a culture. By the use of the model for the increase in numbers of spontaneous mutants on the assumption of 2 generations of segregation lag (Ryan \& Wainwright, 1954; see also Table 2 below), the proportion in the mutant population of mutants which have existed for any given number of generations can be calculated for any population size. In the experiments to be reported, $100 \mathrm{ml}$. cultures of $\mathrm{h}^{-}$bacteria, each containing a total of $c .3 \cdot 7 \times 10^{3} \mathrm{~h}^{+}$ mutants, were used. Among this number of mutants it is calculated that c. $27 \%$ would be less than one generation old and would not increase the population of mutants until two generations of growth had ensued; $13.5 \%$ would be one generation old and would 'lag' for only one generation; the remainder would be out of lag and would grow as rapidly as their parents. Such a mixed population of mutants would be expected to increase less rapidly than a pure culture of mutants homocaryotic for the $\mathrm{h}^{+}$gene, and the extra delay is calculated as $c .0 .5$ generations.

When such mixtures were made, using $\mathrm{h}^{-} \mathrm{lac}^{+}$cultures and added $\mathrm{h}^{+} \mathrm{lac}^{-}$ bacteria and were respread on the surfaces of plates devoid of histidine, the results shown in Fig. 6 were found. The added mutants increased abruptly after a delay of a little more than $1 \mathrm{hr}$. The mutant population, including mutants still in segregation lag, increased gradually with an extra delay of c. $\mathbf{0 . 7}$ generations as calculated from the composite curve or $\mathbf{0 \cdot 6}$ generations when the estimate is the average extra delay found in the individual component experiments. These values were not significantly different by $t$ test 
( $P$ is between 0.5 and 0.6 ) from the 0.5 expected. Thus, once again, the data were consistent with the assumption of an average of four nuclei per bacterium and consequent segregation lag.

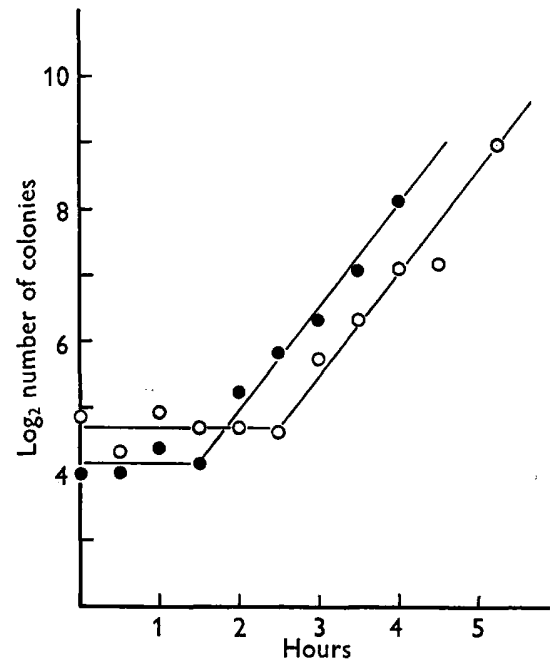

Fig. 5

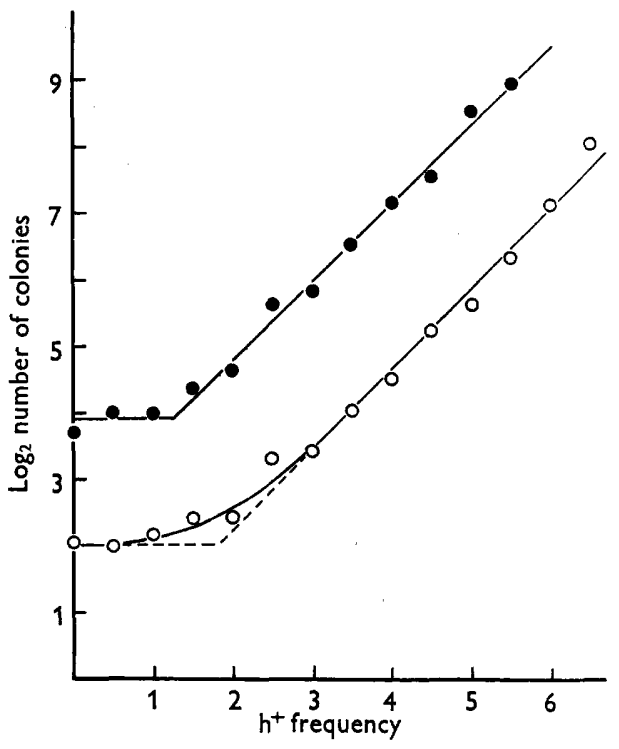

Fig. 6

Fig. 5. The growth of irradiated $h^{+}$lac + bacteria (open circles) spread on the surfaces of agar devoid of histidine to which unirradiated $\mathrm{h}+$ lac - bacteria (black circles) had been added. The ordinate measures the $\log _{2}$ of the number of colonies/plate; the abscissa, the time (hr.) at which the plates were respread. The data are the averages of 3 experiments where the average survival was $2 \cdot 2 \times 10^{-3}$. The generation time during logarithmic growth was $38 \mathrm{~min}$.

Fig. 6. The growth of $\mathrm{h}^{+}$mutants (open circles) of spontaneous origin among unirradiated $h^{-}$lac $^{+}$bacteria spread on the surfaces of agar devoid of histidine and of unirradiated $\mathrm{h}^{+} \mathbf{l a c}^{-}$bacteria (black circles) that had been added to them. The ordinate measures the $\log _{2}$ of the number of colonies/plate; the abscissa, the time (hr.) at which the plates were respread. The data are the averages of 8 experiments where the average spontaneous $h^{+}$mutant frequency was $4.0 \times 10^{-7}\left(\right.$ median $\left.=1.7 \times 10^{-7}\right)$. The generation time during logarithmic growth was 51 min.

\section{DISCUSSION}

The word 'nucleus' has been used throughout this paper as if it were synonymous with 'mutable unit'. The identification of nuclei as the sites of mutation must rest eventually on cytological studies. During the logarithmic phase of growth $\mathrm{h}$ - bacteria seem to have mostly two nuclear bodies/cell. However, there is also a fair-sized class of cells containing what appear to be four nuclear bodies; in addition, there are rare cells containing one, three or more than four bodies and, in our preparations, quite a few bacteria in which the number of nuclear bodies cannot be counted accurately. In view of the difficulty in resolving nuclear bodies in bacteria which have been grown in chemically defined media we cannot be sure that what appear to be single nuclear bodies are not double. It would seem quite possible that newly divided bacteria have two visible nuclear bodies, in each of which the genetic material has already 
duplicated (Robinow, 1945, p. 358). During the growth and elongation of the cell the two bodies separate into four, each of which now has only one set of genetic material. At the time of cell division the genetic material divides and the number of nuclear bodies is reduced to two. The morphological and spatial relationships to be seen among the dividing nuclear bodies would suggest that their separation is regular. If it were irregular the lag in the onset of mutant increase would simply be interrupted giving a gradual average rise at the beginning of the growth curve. The abrupt rise observed speaks for the regularity of nuclear separation. The mutable units, like nuclei, seem to be independent of one another in mutation, to segregate in a regular fashion and to be represented four times on the average in each bacterium.

There are several types of delay that can differentially retard the onset of increase of a mutant population. The more obvious of these can be classified as: (1) delay in cell division; (2) delay in the mutational event; (3) segregation delay; (4) phenotypic delay.

All bacteria exhibit a delay in the onset of division when they are taken from a phase of logarithmic growth into some other and then returned to the original growth-supporting medium. Cultures in the stationary phase or logarithmically growing bacteria that have been washed, show such a physiological delay, but it is usually the same for mutant and parent. It may be longer for the mutant under some conditions such as those induced by a very strong dose of ultraviolet light. In this case the new mutants seem to appear in that fraction of the population which the irradiation has specifically delayed (Ryan, 1954). But doses of this sort, yielding survivals of $c .10^{-5}$, are much greater than those used in the experiments reported above which resulted in survivals of $c .10^{-3}$ and which did not specifically delay the new mutants (Fig. 3). A delay in the mutational event for some time after the application of the inducing stimulus seems to be a real possibility in some instances (Witkin, 1951; Kaplan, 1952; Newcombe, 1953). Such a delay cannot account for the results reported in this paper nor do these data shed any light on the question of mutation delay.

Segregation lag and phenotypic delay are clearly distinguishable in principle and in practice. The former has consequences of the sort described in this paper while the latter, being a delay in the biochemical development of the phenotype, demands that the mutant fail to appear as such until the phenotype is expressed. Thus bacteria possessing an $\mathrm{h}^{+}$genotype would be counted as $\mathrm{h}^{-}$as long as development of the histidine-synthesizing system, which makes the bacterium phenotypically $\mathrm{h}^{+}$, is not completed. As a consequence the frequency of mutants, and in Poisson experiments the fraction of cultures with one or more mutants, would be underestimated. Because when the number of mutants is large the fraction of recent enough origin to be still in phenotypic delay may be small, methods of measuring mutation rate that depend upon the number of phenotypically expressed mutants present will not be as seriously affected by phenotypic delay as methods depending upon the fraction of cultures without mutants. In the latter case, if there were, for example, one generation of phenotypic delay, the fraction of cultures without mutants 
would be that determined by all mutations except those which occurred during the last generation of growth. Since at each generation a number of mutations occur which is, on the average, approximately equal to all preceding mutations (Table 2), the true number of mutations will be underestimated by half. The

Table 2. Expected average increase in the number of mutants on the assumptions of random mutation, no selection and a segregation lag of 2 generations

$\begin{array}{cccc}\begin{array}{c}\text { Generation } \\ 1\end{array} & \begin{array}{c}\text { Parents } \\ n\end{array} & \overbrace{\text { New Old }}^{\text {Mutants }} & \begin{array}{c}\text { Total no. } \\ \text { of mutants } \\ 2\end{array} \\ 3 & 4 n & \begin{array}{c}2+1 \\ 4+2+1\end{array} & 7 \\ 4 & 8 n & 8+4+2+2 & 16 \\ - & - & --2- & -\end{array}$

total number of bacteria, on the other hand, is correctly determined. Consequently the mutation rate, $a$, will be underestimated by half for every generation of phenotypic lag when the formula is used that defines mutation rate

$$
a=\frac{m}{N} \ln 2,
$$

where $N / \ln 2$ is the total number of bacteria-generations that have existed during the growth of the culture and $m$ is the average number of mutations, based upon the proportion of cultures without mutants and on the zero term of the Poisson formulation, $\boldsymbol{P}_{0}=\mathrm{e}^{-m}$.

In the first specific study of phenotypic lag following bacterial mutation, Newcombe (1948) explained on that basis the low rate of mutation of Escherichia coli to resistance to bacteriophage $\mathrm{T}_{1}$ when determined by the Poisson method as compared with methods based upon the numbers of mutants present in different cultures. But the latter methods assume that the distribution of the numbers of mutants is in accord with the assumptions of random mutation, equal growth of parent and mutant and no segregation lag. If these assumptions are correct, the distribution of $T_{1}$ resistant mutants should be that calculated by Lea \& Coulson (1949); but it is not (Ryan, 1952): Consequently, mutation rates calculated on the basis of numbers of mutants are unreliable and discrepancies between them and rates calculated from the fraction of cultures without mutants do not demonstrate the operation of phenotypic lag. Furthermore, working with mutations to streptomycin resistance, which is a recessive character in $\boldsymbol{E}$. coli (Lederberg, 1951) and one whose distribution among cultures would not be expected to be, and was not observed to be, affected by segregation lag, Newcombe \& Hawirko (1949) found no discrepancy between mutation rates calculated by the two types of methods. Hence, mutation rate comparisons have not yet yielded evidence of phenotypic lag. 
Whenever phenotypic lag lasts for more than a generation no new mutants can appear until growth has ensued. Demerec (1946) has interpreted in terms of phenotypic lag the failure to find induced phage-resistant mutants until after a period of growth had intervened. This and the delayed appearance of mutants in general may, however, have other interpretations (Ryan, 1954). The contention of Lieb (1951) that ultraviolet-induced $\mathrm{h}^{+}$mutants do not express themselves without a period of intermediate cultivation has not been confirmed (Kaplan, 1950; Ryan, 1954). Working with other mutations to prototrophy, Davis (1950) found that after treatment with ultraviolet light the number of prototrophic mutants was increased when a small amount of the growth factor required by the parents was added to the cultures. The role of the added growth factor is not clear in this case and, as a consequence, neither is the role of phenotypic lag. There must, of course, be some time required for phenotypic expression but it is a question whether this time is as great as one or more generations.

Segregation lag and phenotypic delay could very well operate simultaneously. If the mutation involved were 'recessive' in a heterocaryon, phenotypic delay would begin after the mutant became homocaryotic for mutant nuclei. Thus, the mutant could not express itself without previous growth. If, on the other hand, the mutation were 'dominant' in a heterocaryon, phenotypic delay would begin at the moment of mutation. If the development of the phenotype required, for example, one generation and there were two generations of segregation lag, growth would not take place in the absence of the growth factor required by the parent; hence, the mutant would not be detected. If the growth factor were supplied, however, development of the phenotype could be achieved. But during this period, until the end of which the mutant would be cryptic, one generation of segregation lag would have occurred. From the time of appearance of the mutant only one generation of segregation delay would be observed. Thus a generation of phenotypic delay would conceal one generation of segregation and the mutation rate, determined from the frequency of cultures without mutants would be halved by each generation of phenotypic delay. These points can be seen perhaps more clearly if one imagines that all of the new mutants listed in the appropriate column in Table 2 are in phenotypic lag and hence are cryptic.

Thus, induced $\mathrm{h}^{+}$mutants, on any assumption of phenotypic delay, would not be expected to be detectable without growth, and yet they are (Ryan, 1954). This fact is most clearly shown in Fig. 2 of this paper. After irradiation, bacteria of $\mathrm{h}^{-}$phenotype not only fail to grow, but die on agar devoid of histidine; yet new $\mathrm{h}^{+}$mutants express themselves and grow normally-they are zero point mutants. Therefore, no division is needed for the expression of the $\mathrm{h}^{+}$phenotype. It can be further concluded that the $\mathrm{h}^{+}$mutation is dominant in heterocaryons on the following argument. The development of the $h^{+}$phenotype is required for growth on agar devoid of histidine. On such agar induced $h^{+}$mutants do appear and multiply while bacteria of $h^{-}$phenotype do not. Therefore, the $\mathrm{h}^{+}$phenotype must have been imposed without previous division. Since there is a delay in the initiation of the mutant clone 
due to the segregation of nuclei, the newly mutated $\mathrm{h}^{+}$gene must have influenced the $\mathrm{h}^{+}$phenotype even in the presence of its $\mathrm{h}^{-}$sisters which were later segregated. Therefore, it was dominant in a heterocaryon.

It might be supposed that, in the heterocaryons formed upon mutation, the $\mathrm{h}^{+}$gene is recessive but that the three sister $\mathrm{h}^{-}$nuclei were inactivated by irradiation (and also, perhaps, by the process leading to the death of $h$ - bacteria observed in Fig. 2). Thus, the cells would be effectively uninucleate and hence homocaryotic for the $\mathrm{h}^{+}$nuclei. The observed segregation delay would involve the separation of the active $h^{+}$nucleus from its inactivated $h^{-}$sisters. But this hypothesis of $\mathrm{h}^{+}$recessivity would, further, have to assume that along with inactivation of $\mathrm{h}^{-}$nuclei there was inactivation of all parts of the suppressive $\mathrm{h}^{-}$phenotype. Furthermore, since delay in the onset of mutant increase does not take place when a mutation is recessive (assume in Table 2 that all new mutants are cryptic for two generations), this hypothesis will not account for the lag of spontaneous $\mathrm{h}^{+}$mutants occurring among unirradiated $\mathrm{h}$ - bacteria which neither die nor grow when spread on agar devoid of histidine.

This latter fact was attested by six experiments in which an average of $5 \cdot 4 \times 10^{4}$ washed unirradiated $h^{-}$bacteria were spread on agar plates devoid of histidine and washed off again at various times up to $24 \mathrm{hr}$. There was a slight increase that ceased at a level of $7 \cdot 2 \times 10^{4}$ bacteria/plate. Thus, the agar could support the division of only $c .2 \times 10^{4}$ among the $c .2 \times 10^{7} \mathrm{~h}^{-}$bacteria spread on the plates used in the spontaneous $h^{+}$experiments. The chance that a heterocaryon, still phenotypically $h^{-}$, could divide is therefore only $10^{-3}$ at a maximum. Since the frequency of $h^{+}$mutants recovered in medium with histidine (Ryan, 1954) is the same as that obtained in its absence, the very slight growth in the absence of added histidine could not mask a possible recessive nature of the $\mathrm{h}^{+}$gene.

A final argument regarding the role of phenotypic lag in the $\mathrm{h}^{-} \rightarrow \mathrm{h}^{+}$system can be made as follows. From data on the spontaneous mutation it has been established that the length of segregation lag minus phenotypic lag is $c .2$ generations (Ryan \& Wainwright, 1954). Therefore, segregation lag has a minimum length of $c .2$ generations. If the h- mutable units respond to ultraviolet light independently, then the delay between irradiation and the onset of exponential growth of the induced $h^{+}$mutant clone gives the delay due to segregation lag plus phenotypic lag. Hence, it established the maximum length of segregation lag also as $c .2$ generations. By difference, phenotypic lag must be short, and lasts less than 1 generation.

The simplest conception, consistent with all available data, portrays the $h^{-}$bacterium as possessing, on the average, four mutable $h^{-}$genes. Mutation to the $\mathrm{h}^{+}$condition is spatially random and results in the formation of a heterocaryon. In this condition the newly mutated $\mathrm{h}^{+}$gene is able to evoke the development of the $\mathrm{h}^{+}$phenotype in a short period of time and without growth. Once the $\mathrm{h}^{+}$phenotype is developed growth occurs even in the absence of histidine. During this growth the $h^{-}$genes segregate regularly and after two divisions a cell homocaryotic for $\mathrm{h}^{+}$nuclei is formed. Only then does the $\mathrm{h}^{+}$mutant clone increase normally by doubling. 
This work was supported in part by an American Cancer Society grant recommended by the Committee on Growth and by a research grant from the National Institutes of Health, U.S. Public Health Service.

\section{REFERENCES}

Davis, B. D. (1950). Studies on nutritionally deficient bacterial mutants isolated by means of penicillin. Experientia, 6, 41 .

Demerec, M. (1946). Induced mutations and possible mechanisms of the transmission of heredity in Escherichia coli. Proc. nat. Acad. Sci., Wash. 32, 36.

Demerec, M. \& CahN, E. (1953). Studies of mutability in nutritionally deficient strains of Escherichia coli. 1. Genetic analysis of five auxotrophic strains. J. Bact. 65, 27.

Kaplan, R. W. (1950). Mutation und Keimtötung bei Bact. coli histidinless durch UV und Photodynamie. Naturwissenschaften. 23, 547.

KaPLAN, R. W. (1952). Auslösung von Farbsektor- und anderen Mutationen bei Bacterium prodigiosum durch monochromatisches Ultra violett verschiedener Wellenlängen. $Z$. Naturf. $7 b, 291$.

LEA, D. E. \& Coulson, C. A. (1949). The distribution of the numbers of mutants in bacterial populations. J. Genet. 49, 264.

Lederberg, J. (1951). Dominance of streptomycin sensitivity in $E$. coli heterozygotes. Microbial Genetics Bulletin 4, 14.

Lederberg, J. \& Lederberg, E. M. (1952). Replica plating and indirect selection of bacterial mutants. J. Bact. 63, 399.

LiEB, M. (1951). Forward and reverse mutation in a histidine-requiring strain of Escherichia coli. Genetics, 36, 460.

Newcombe, H. B. (1948). Delayed expression of spontaneous mutations in Escherichia coli. Genetics, 33, 447.

Newcombe, H. B. (1953). The delayed appearance of radiation induced genetic change in bacteria. Genetics, 38, 134.

Newcombe, H. B. \& Hawirko, R. (1949). Spontaneous mutation to streptomycin resistance and dependence in Escherichia coli. J. Bact. 57, 565.

Newcombe, H. B. \& Scott, G. W. (1949). Factors responsible for the delayed appearance of radiation-induced mutants in Escherichia coli. Genetics, 34, 475.

RoBiNow, C. F. (1945). Nuclear apparatus and cell structure in rod-shaped bacteria. Addendum in Dubos, R. J., The Bacterial Cell, Cambridge, Mass.: Harvard University Press.

RYAN, F. J. (1952). Distribution of numbers of mutant bacteria in replicate cultures. Nature, Lond., 169, 882.

Ryan, F. J. (1954). The delayed appearance of mutants in bacterial cultures. Proc. Nat. Acad. Sci., Wash. 40, 178.

Ryan, F. J. \& WAINwright, L. K. (1954). Nuclear segregation and the growth of clones of spontaneous mutants of bacteria. J. gen. Microbiol. 11, 364.

Wiтkin, E. (1951). Nuclear segregation and the delayed appearance of induced mutants in Escherichia coli. Cold Spr. Harb. Sym. quant. Biol. 16, 357.

(Received 26 April 1954) 